\title{
On a Theorem of Ziv Ran concerning Abelian Varieties Which Are Product of Jacobians
}

\author{
Cristian Anghel and Nicolae Buruiana \\ Department of Mathematics, Institute of Mathematics of the Romanian Academy, Calea Grivitei No. 21, 010702 Bucuresti, Romania
}

Correspondence should be addressed to Cristian Anghel; cristian.anghel@imar.ro

Received 21 July 2015; Accepted 21 September 2015

Academic Editor: Kaleem R. Kazmi

Copyright ( 2015 C. Anghel and N. Buruiana. This is an open access article distributed under the Creative Commons Attribution License, which permits unrestricted use, distribution, and reproduction in any medium, provided the original work is properly cited.

We give a new proof for a theorem of Ziv Ran which generalizes some results of Matsusaka and Hoyt. These results provide criteria for an Abelian variety to be a Jacobian or a product of Jacobians. The advantage of our method is that it works in arbitrary characteristic.

\section{Introduction}

In the classical papers [1] (resp. [2]), Matsusaka and Hoyt gave a necessary and sufficient criterion for an Abelian variety $A$ for being a Jacobian, respectively, a product of Jacobians. In [3], Ran reconsiders the subject and gives a more general and probably more natural criterion for this. However, his method seems unsatisfactory in positive characteristic.

The aim of this paper is to reprove Ran's criterion, using results from [4] on the ring of the numerical algebraic cycles on $A$. For the particular case of the Ran-Matsusaka criterion, another proof appeared in [5]. Both proofs are independent of the characteristic of the base field.

In the sequel, for an Abelian variety $A$, we denote by $Z(A)$ the $\mathbb{Q}$-vector space of the algebraic cycles with $\mathbb{Q}$-coefficients on $A$ and by $N(A)$ the quotient by numerical equivalence. It is well known (cf. [6] or [4]) that on $N(A)$ there are two $\mathbb{Q}$-algebra structures given by the usual product and by the Pontrjagin product.

Throughout this paper, the latter will be very useful thanks to its geometric definition and to the fact that it gives a ring structure not only on $N(A)$ but also on $Z(A)$. Below, for $x, y \in N(A)$, we will denote the usual product by $x \cdot y$ and the Pontrjagin product by $x \star y$.

Also, for the two subvarieties $X_{1}$ and $X_{2}$ in $A$, we denote by $X_{1} \dot{+} X_{2}$ and $X_{1}-X_{2}$ their sum and difference in the group law of $A$, to avoid confusion with the corresponding operations on the cycles. In the current paper, the algebraic cycles will often be divisors and 1-cycles (the latter ones being formal sums of curves) and they will always have integer coefficients.

The term curve, is reserved for integral ones, and all 1cycles will be considered effective. Finally, a prime cycle is an irreducible subvariety of $A$ of a corresponding codimension.

\section{Generating Curves on an Abelian Variety}

Let $A$ be an Abelian variety of dimension $n$ and $E$ be a curve on it containing the origin $0_{A}$ of $A$. We consider a sequence of closed subsets in $A$, defined as follows:

$$
\begin{aligned}
E_{0} & =\left\{0_{A}\right\} \\
E_{i} & =E \dot{+} E \dot{+} \cdots \dot{+} E \quad(i \text { terms, with } 1 \leq i \leq n), \\
E_{n+1} & =A .
\end{aligned}
$$

It is clear that this sequence is increasing, $\operatorname{dim} E_{i} \leq i$ and $\operatorname{dim} E_{i+1} \leq \operatorname{dim} E_{i}+1$ for every $i$. As long as $E$ is a curve, $E_{i}$ is irreducible and there is a first index $j$ such that $E_{j}=E_{j+1}$. Also, we have $E_{i}=E_{j}$ for all $i \geq j$ and $\operatorname{dim} E_{j}=j$. It follows that $E_{j}$ is stable for the group law on $A$ and the induced operation has $0_{A}$ as unity. Using a result of Ramanujan in ( [7, chapter II, Section 4]), $E_{j}$ is an Abelian subvariety of $A$ and the points of $E$ generate the group $E_{j}$. We denote by $\langle E\rangle$ 
the subvariety $E_{j}$. If $j=n$, then $\langle E\rangle=A$ and we say that $E$ is a generating curve on $A$.

Remark 1. In [8] Matsusaka proves that every Abelian variety has a generating curve. Moreover, from his proof, for a projective embedding of $A$, every linear section with a convenient linear subspace of appropriate dimension which contains $0_{A}$ is a generating curve for $A$.

Using the Pontrjagin product (for cycles, not for numerical classes) it is easy to deduce the following useful fact.

Lemma 2. Let $E$ be a curve in $A$ with $0_{A} \in E,\langle E\rangle$ the subvariety of $A$ generated by $E$ and $j=\operatorname{dim}\langle E\rangle$. Then, $j$ is the maximal number $i$ such that $E^{* i}(=E * E * \cdots * E$ with $i$ terms) is nonzero and $\langle E\rangle$ is the support of the cycle $E^{* j}$.

Let us now consider a curve $E \subset A$ which does not necessarily contain the origin. It easily follows that for $x \in E$ the Abelian variety generated by $E-\{x\}$ does not depend on $x$; it is in fact the subgroup of $A$ generated by $E-E$. This Abelian variety will also be denoted by $\langle E\rangle$. If $Z=m_{1} E_{1}+\cdots+m_{k} E_{k}$ is an effective 1-cycle, we denote by $\langle Z\rangle$ the Abelian subvariety given by $\left\langle E_{1}\right\rangle \dot{+}\left\langle E_{2}\right\rangle \dot{+} \cdots \dot{+}\left\langle E_{k}\right\rangle$ and we will say that $Z$ is a generating 1 -cycle for $\langle Z\rangle$.

Remark 3. From the definition above, we see that the construction of $\langle Z\rangle$ is independent of the numbers $m_{i}$. In particular, $Z$ and $Z_{\text {red }}$ generate the same subvariety and also for $Z$ and $m Z$.

The next lemma will be useful in the sequel.

Lemma 4. (a) For $Y, E \subset A$ with $Y$ subvariety and $E$, a curve, both containing the origin, if $Y * E=0$ then $E \subset Y$.

(b) For $Y_{1}, Y_{2} \subset A$ Abelian subvarieties and $m, n$ nonzero integers, if $m Y_{1}$ and $n Y_{2}$ are numerically equivalent, then $Y_{1}=$ $Y_{2}$.

Proof. (a) We have $\operatorname{dim} Y \leq \operatorname{dim}(Y \dot{+} E) \leq 1+\operatorname{dim} Y$ and from $Y * E=0$ we deduce that $\operatorname{dim}(Y \dot{+} E)<1+\operatorname{dim} Y$. So $Y=Y \dot{+} E$ and because $0_{A} \in Y$ it follows $E \subset Y$.

(b) Let $E_{1}$ be a generating curve for $Y_{1}$ (it exists cf. Remark 1). Then $m Y_{1} * E_{1}=m\left(Y_{1} * E_{1}\right)=0$ and so $n Y_{2} * E_{1}=n\left(Y_{2} * E_{1}\right)=0$. It follows that $Y_{2} * E_{1}=0$, and from the first point $E_{1} \subset Y_{2}$. The last inclusion implies that $Y_{1}=\left\langle E_{1}\right\rangle \subset Y_{2}$. In the same way the reversed inclusion is proved.

Remark 5. The point (b) above, in the case $m=n=1$, is a result of Matsusaka in [8].

Proposition 6. (a) For two curves $E_{1}, E_{2} \subset A$ which are numerically equivalent, we have $\left\langle E_{1}\right\rangle=\left\langle E_{2}\right\rangle$.

(b) For a curve $E$ and $Z$, a 1-cycle which is numerically equivalent with $E$, we have $\langle Z\rangle=\langle E\rangle$.

(c) Let $D$ be an ample divisor and $Z$ a 1-cycle which is numerically equivalent with $D^{n-1}$. Then $Z$ is a generating $1-$ cycle for $A$.
Proof. (a) Using convenient translations we can suppose that $E_{1}$ and $E_{2}$ contain $0_{A}$. Let $r, s$ be the dimensions of $Y_{i}=$ $\left\langle E_{i}\right\rangle$ for $i=1,2$. Using Lemma 2 and the fact that $E_{1}{ }^{* a}$ is numerically equivalent with $E_{2}{ }^{* a}$ for all positive integers $a$, we find $r=s$. From Lemma 2 again, $E_{1}{ }^{* r}$ is a multiple of both $Y_{1}$ and $Y_{2}$, and the conclusion follows from Lemma 4(b).

(b) As in (a), denoting $Z=m_{1} E_{1}+\cdots+m_{k} E_{k}$ we can suppose that $E$ and all $E_{i}$ contain $0_{A}$. By Lemma 2, for $r=\operatorname{dim}\langle E\rangle$, we have $E^{*(r+1)}=0$. Now, $Z^{* r}$ being numeric equivalent with $E^{* r}$, we find $Z^{* r} * E=0$. But again from Lemma 2 we find a nonzero term in the development of $Z^{* r}$. With Lemma 4(a), this term which is in fact a subvariety contains $E$, because all terms in $Z^{* r}$ are vanished by the Pontrjagin product with $E$. On the other hand, this term is contained in $\langle Z\rangle$ and so $\langle E\rangle \subset\langle Z\rangle$.

For the reverse inclusion, we consider the development of the left side of the equality $Z * E^{* r}=0$. From Lemma $2 E^{* r}=$ $n\langle E\rangle$ with $n \geq 1$ is an integer. We find $m_{1} n E_{1} *\langle E\rangle+\cdots+$ $m_{k} n E_{k} *\langle E\rangle=0$; therefore $E_{i} *\langle E\rangle=0$ for all $i$ and then from Lemma 4(a), $E_{i} \subset\langle E\rangle$, so $\langle Z\rangle \subset\langle E\rangle$.

(c) Let $m$ be a positive integer with $|m D|$ very ample. The cycles $m^{n-1} D^{n-1}$ and $m^{n-1} Z$ are therefore numerical equivalents and there will exist an integer curve $E$ in the same numeric class with $m^{n-1} D^{n-1}$ and so with $m^{n-1} Z$. From (b) we have $\langle E\rangle=\left\langle m^{n-1} Z\right\rangle=\langle Z\rangle$. But from Remark $1\langle E\rangle=A$, so $Z$ is a generating 1 -cycle.

The point (c) above is a slight generalization of the result from Remark 1 and will be used to deduce the MatsusakaHoyt criterion from that of Ran.

\section{Algebraic Cycles Constructed from Generating Curves}

We recall a result from [4] which will be the main tool in the proof of Ran's theorem. Let $E$ be a generating curve of the $n$-dimensional Abelian variety $A$. We consider on $A$ the following cycles: $W_{n}(E)=\left\{0_{A}\right\}$ and $W_{i}(E)=(1 /(n-$ $i) !) E^{*(n-i)}$ for $0 \leq i \leq n-1$. From the definition of the Pontrjagin product, $W_{i}(E)$ is a cycle with irreducible support of codimension $i$ on $A$. In particular $W_{1}(E)$ is a divisor and there exists $\alpha_{E} \in \mathbb{Q}$ such that $W_{0}(E)=\alpha_{E} \cdot 1_{A}$, where $1_{A}$ is the fundamental cycle on $A$.

The result we need from [4] is the following.

Proposition 7. All cycles $W_{i}(E)$ have integer coefficients and in particular $\alpha_{E} \in \mathbb{Z}$, being evidently positive. Also, $W_{1}(E)^{i}=$ $i ! \alpha_{E}{ }^{i-1} W_{i}(E)$ for $1 \leq i \leq n$. In particular, $W_{1}(E)^{n}>0$ and so $W_{1}(E)$ is ample.

Remark 8. For $E$ a smooth curve and $A$ its Jacobian, these divisors are well-known.

A first application of the proposition above is point (b) in the following.

Proposition 9. (a) Let $D$ be an effective divisor and $Z$ a generating 1 -cycle. Then $D \cdot Z>0$.

(b) If moreover $D$ is ample, then $D \cdot Z \geq n=\operatorname{dim} A$. 
Proof. (a) We can suppose that $D$ is a prime divisor. Let $E_{1}, \ldots, E_{k}$ be the components of $Z$. We have $D \cdot E_{i} \geq 0$ for all $i$, because the general translation of $E_{i}$ cuts properly $D$. It is therefore sufficient to find an $i$ such that $D \cdot E_{i}>0$. Suppose there is no such $i$. Then using a result from [7, chapter2, Section 6] translations with elements of the form $\{x\} \cdot\{y\}$ with $x, y \in E_{i}$ leave $D$ invariant. But $Z$ is a generating 1 -cycle, and therefore every element in $A$ is a sum of elements of this form. So $D$ is invariant with respect to any translation and then numerically equivalent with 0 , in contradiction with its effectiveness.

(b) Consider a first case where $Z=E$ is a prime cycle (i.e., $E$ is a curve) and without loss of generality $0_{A} \in E$. Let $t$ be a variable and consider the polynomial $P(t)=\left(t \cdot W_{1}(E)+\right.$ $D)^{n}=n ! \alpha_{E}{ }^{n-1} t^{n}+n ! \alpha_{E}{ }^{n-2}(D \cdot E) t^{n-1}+\cdots+D^{n}$. Because $W_{1}(D)$ is ample and $D$ is nondegenerate, the index theorem for Abelian varieties compare [7] asserts that all roots of $P$ are real and negative. So the means inequality gives $D \cdot E \geq$ $n\left(\chi\left(\mathcal{O}_{A}(D)\right) \cdot \alpha_{E}\right)^{1 / n} \geq n$.

For the general case, let $Z=m_{1} E_{1}+\cdots+m_{k} E_{k}$ with all $m_{i}>0, X_{i}=\left\langle E_{i}\right\rangle$, and $D_{i}$ be the restriction of $D$ to $X_{i}$. The projection formula gives $D \cdot E_{i}=D_{i} \cdot E_{i} \geq \operatorname{dim} X_{i}$ from the particular case above. So, $D \cdot Z=\sum_{i} m_{i} D \cdot E_{i} \geq \sum_{i} D$. $E_{i} \geq \sum_{i} \operatorname{dim} X_{i} \geq \operatorname{dim} A=n$, because $Z$ is a generating 1cycle.

The following consequence of the above proposition will be useful in the last part of the paper.

Corollary 10. Let $A$ be an Abelian variety, $D=\sum_{i=1}^{r} m_{i} D_{i}$ an ample effective divisor, and $Z=\sum_{i=1}^{s} n_{j} E_{j}$ a generating 1-cycle of $A$ (the coefficients are supposed to be nonzero). If $D \cdot Z=$ $n=\operatorname{dim} A$ then $m_{i}=n_{j}=1$ for all $i, j$.

Proof. We have $n=D \cdot Z=\sum_{i=1}^{r} m_{i} D_{i} \cdot Z \geq \sum_{i=1}^{r} D_{i} \cdot Z \geq n$ because $\sum_{i=1}^{r} D_{i}$ is ample and one can apply Proposition 9(b). So $m_{i} D_{i} \cdot Z=D_{i} \cdot Z$ and because the last term is nonzero by Proposition 9(b), we find $m_{i}=1$ for all $i$.

In the same way, $n=D \cdot Z=\sum_{i=1}^{s} n_{j} D \cdot E_{j} \geq \sum_{i=1}^{s} D \cdot E_{j}=$ $D \cdot \sum_{i=1}^{s} E_{j} \geq n$ because $\sum_{i=1}^{s} E_{j}$ remains a generator 1-cycle by Remark 3. So $n_{j} D \cdot E_{j}=D \cdot E_{j}$ and $D$ being ample, the last term is positive. It results that $n_{j}=1$ for all $j$.

We can now prove the following result, which is nothing else but Ran's version of the Matsusaka theorem.

Theorem 11. Let $D$ be an ample divisor on the Abelian variety $A$ and let $E$ be a generating curve such that $D \cdot E=n=\operatorname{dim} A$. Then $E$ is smooth, $A$ is its Jacobian, and $D$ is a translation of $W_{1}(E)$.

Proof. In the proof of point (b) from Proposition 9 we have the inequality $D \cdot E \geq n\left(\chi\left(\mathcal{O}_{A}(D)\right) \cdot \alpha_{E}\right)^{1 / n} \geq n$. If $D \cdot E=n$ we will have $\chi\left(\mathcal{O}_{A}(D)\right)=\alpha_{E}=1$ and so $D^{n}=n$ !. In this case the polynomial $P(t)$ from the same proposition becomes $P(t)=n ! t^{n}+n ! \cdot n \cdot t^{n-1}+\cdots+n !$. It follows that the arithmetic and geometric means of the roots coincide and so all the roots have the form $-\lambda$ for a positive value of $\lambda$. So $P(t)=n !(t+\lambda)^{n}$ and by identification, $\lambda^{n}=1$. It follows that $\lambda=1$ and then
$W_{1}(E)^{n-1} \cdot D=W_{1}(E)^{n-2} \cdot D^{2}=n !$. These relations imply that $\left(D-W_{1}(E)\right) \cdot W_{1}(E)^{n-1}=\left(D-W_{1}(E)\right)^{2} \cdot W_{1}(E)^{n-2}=0$. The Hodge index theorem asserts that $D$ is numerically equivalent with $W_{1}(E)$, and because $W_{1}(E)$ is a principal polarization (from Proposition 7 and equality $\alpha_{E}=1$ ), one may deduce that $D$ is a translation of $W_{1}(E)$.

Consider the normalization $f_{0}: T \rightarrow E$ for $E$, and let $f: J \rightarrow A$ be a prolongation of $f_{0}$, where $J$ is a Jacobian of $T$. If we choose a base point in the construction of $J$, one on $T$ which sits above $0_{A} \in E, f$ will be a morphism of Abelian varieties, sending origin to origin. Also, $f$ is surjective because $E$ is generating for $A$ and for $g=$ genus of $T$ we have $g \geq n$.

Let us denote by $W_{i}=W_{i}(T)$ the canonical cycles on the Jacobian $J$. Therefore $f_{*}\left(W_{g-i}\right)=W_{n-i}(E)$ for $1 \leq i \leq n$ : for $i=1$ this is clear because $W_{g-1}=T$ and for $i>1$ it is a consequence of the definitions for $W_{g-i}$ and $W_{g-i}(E)$ and also from the fact that $f_{*}$ commute with the Pontrjagin product. In particular $f_{*}\left(W_{g-n}\right)=W_{0}(E)=\alpha_{E} \cdot 1_{A}$ and so $\alpha_{E}=1$ is the degree of the restriction of $f$ to $W_{g-n}$. Therefore this restriction is a birational morphism and has an inverse: $A---\rightarrow W_{g-n}$. This inverse, considered as a rational map from $A$ to $J$ can be extended over all the $A$ giving a morphism $A \rightarrow J$ compare [7]. As a consequence, the restriction $g$ of $f$ to $W_{g-n}$ will be an isomorphism and $W_{g-n}$ will be an Abelian subvariety of $J$. But $W_{g-n}$ contains $W_{g-1}=T$ which generates $J$ and so $W_{g-n}=J$. In this case we have $g=n$ and $f$ is birational from $J$ to $A$ hence an isomorphism.

\section{Proof of Ran Theorem}

The purpose of this section is to give a proof for Ran's full theorem. Some points are as in [3] and are included only for the sake of completeness. The modifications appear from the replacement of Lemma II.8 from [3] with the result below whose proof is very simple.

Lemma 12. Let $D$ be a prime divisor on an Abelian variety $A$. Then, there exists an Abelian variety $B$, a surjective morphism of Abelian varieties $f: A \rightarrow B$ and an ample divisor $F$ on $B$ such that $f^{-1}(F)=D$ as schemes.

Proof. We consider the closed subgroup $K$ of $A$ defined by $K:=\{x \in A \mid\{x\}+D=D\}$ and the Abelian subvariety $K_{0}$ of $A$ which is the connected component of $0_{A}$ in $K$. We denote by $B$ the quotient $A / K_{0}$ and by $f: A \rightarrow B$ the quotient morphism. Finally we denote by $F$ the closed irreducible subset $f(D)$ with the reduced structure. We easily find $\operatorname{dim} F=\operatorname{dim} B-1$, so $F$ is a divisor on $B$ and set theoretically $f^{-1}(F)=D \dot{+} K_{0}=D$ because $K_{0} \subset K$. Let $x \in A$ such that $\{f(x)\} \dot{+} F=F$. Applying $f^{-1}$ we find $\{x\} \dot{+} D \dot{+} K_{0}=$ $D \dot{+} K_{0}$, and because $D \dot{+} K_{0}=D$ we find $\{x\} \dot{+} D=D$ and so $x \in K$. Therefore, the elements in $B$ which leave $F$ invariant by translations are from $f(K)$. They are then in a finite number, because the index $\left[K: K_{0}\right]$ is finite. So $F$ is an ample divisor on $B$. Finally the equality $f^{-1}(F)=D$ also holds at the schemes level, because $f$ is smooth from its construction. 

Ran.

The result we are interested in is the following theorem of

Theorem 13. Let $A$ be an Abelian variety of dimension $n$, $D=\sum_{i=1}^{r} m_{i} D_{i}$ an ample effective divisor, and $Z=\sum_{j=1}^{s} n_{j} E_{j}$ a generating 1-cycle such that $D \cdot Z=n$. Then $m_{i}=n_{j}=1$ for all $i, j, r=s$ and there are $r$ smooth curves $T_{1}, \ldots, T_{r}$ with Jacobians $B_{1}, \ldots, B_{r}$ and an isomorphism of Abelian varieties $h: B_{1} \times \cdots \times B_{r} \rightarrow A$ such that, for every $i, E_{i}$ is a translation of $h\left(\{0\} \times \cdots \times\{0\} \times T_{i} \times\{0\} \times \cdots \times\{0\}\right)\left(T_{i}\right.$ on the ith place $)$ and $D_{i}$ is a translation of $h\left(B_{1} \times \cdots \times B_{i-1} \times W_{i} \times B_{i+1} \times \cdots \times B_{r}\right)$, where $W_{i}$ is the canonical divisor $W_{1}\left(T_{i}\right)$ on $B_{i}$.

Proof. The fact that $m_{i}=n_{j}=1$ for all $i, j$ is Corollary 10. For the other points, the proof follows closely the one from [3] with some modifications of the arguments. We began with three preliminary steps.

Step 1 . We prove that for every $j$ there is a unique $i$ such that $D_{i} \cdot E_{j} \neq 0$. We translate the curves $E_{j}$ such that they contain the origin and denote the result with the same letter. Let $A_{j}=$ $\left\langle E_{j}\right\rangle$ and $d_{j}=\operatorname{dim} A_{j}$, so that $E_{j}$ is a generating curve for $A_{j}$. Denote by $e_{j}$ the inclusion $A_{j} \hookrightarrow A$ and by the same letter $D$ a translation of the divisor $D$ which has a proper intersection with every $A_{j}$. Therefore, $e_{j}{ }^{*}(D):=D_{j}^{\prime}$ is defined as a cycle and is an ample divisor on $A_{j}$. The projection formula gives

$$
D \cdot E_{j}=D_{j}^{\prime} \cdot E_{j}
$$

and so

$$
n=D \cdot E=\sum_{j=1}^{s} D \cdot E_{j}=\sum_{j=1}^{s} D_{j}^{\prime} \cdot E_{j} \geq \sum_{j=1}^{s} d_{j} \geq n .
$$

The first inequality comes from the fact that on $A_{j}$ one has $D_{j}^{\prime}$. $E_{j} \geq d_{j}$ according to Proposition 9(b), and the last one is due to the fact that $Z$ is a generating 1 -cycle. So $D_{j}^{\prime} \cdot E_{j}=d_{j}$, and $E_{j}$ being a generating curve for $A_{j}$, from Theorem 11 one finds that $E_{j}$ is smooth, $A_{j}$ is its Jacobian, and $D_{j}^{\prime}$ is a translation of the canonical divisor on $A_{j}$; so $D_{j}^{\prime}$ is prime as any divisor numeric equivalent with it (it is a principal polarization).

Let us fix $j$, and consider for any $i$ a translation of $D_{i}$ which cuts $A_{j}$ properly. Every such translation, also denoted by $D_{i}$, restricted to $A_{j}$ either is an effective divisor or has an empty intersection with $A_{j}$, in which case $D_{i} \cdot E_{j}=0$. But the sum of these restrictions is numerically equivalent with $D_{j}^{\prime}$ and so there cannot be two indexes $i$ with $D_{i} \cdot E_{j} \neq 0$, because in such a case $D_{j}^{\prime}$ which is prime would be the sum of two effective divisors. The existence of an $i$ with $D_{i} \cdot E_{j} \neq 0$ comes from the fact that $D$ is ample.

Step 2. This part consists in the proof of the following fact: for an $n$-dimensional Abelian variety $A$, a prime ample divisor $D$, and a generating 1 -cycle $Z=T_{1}+\cdots+T_{s}$ with $D \cdot Z=n$ one has $s=1$ (i.e., $Z$ is ireducible and reduced).

The proof is due to Ran compare Lemma III.2 from [3]. Denote by $A_{1}=\left\langle T_{1}\right\rangle$. From the first step, we know that $A_{1}$ is in fact the Jacobian of the smooth curve $T_{1}$; in particular it is principally polarized and isomorphic with its dual. It will suffice to prove that $A_{1}=A$, because in this case $T_{1}$ will be a generating curve, and the fact that $D$ is ample together with the inequalities $n \leq D \cdot T_{1} \leq D \cdot Z=n$ implies that $s=1$ as desired.

For the time being, we replace $D$ with a translation whose restriction $D_{\mid A_{1}}:=D_{1}$ is well defined as divisor on $A_{1}$. As in the proof of Step $1, D_{1}$ is numerically equivalent with $W_{1}\left(T_{1}\right)$. Let $s: A \times A_{1} \rightarrow A$ the morphism given by $s(r, y)=r+$ $y$ and $p, p_{1}$ be the projections. Consider on $A \times A_{1}$ the line bundle $\mathscr{M}=s^{*}\left(\mathcal{O}_{A}(D)\right) \otimes p_{1}{ }^{*}\left(\mathcal{O}_{A_{1}}\left(-D_{1}\right)\right)$ and on $A_{1} \times A_{1}$ the line bundle $\mathscr{P}=\left(s_{\mid A_{1} \times A_{1}}\right)^{*}\left(\mathcal{O}_{A_{1}}\left(D_{1}\right)\right) \otimes q_{1}{ }^{*}\left(\mathcal{O}_{A_{1}}\left(-D_{1}\right)\right) \otimes$ $q_{2}{ }^{*}\left(\mathcal{O}_{A_{1}}\left(-D_{1}\right)\right)$, where $q_{1}, q_{2}$ are the projections on the factors of $A_{1} \times A_{1}$. Using the fact that $A_{1}$ is a Jacobian (and therefore it is its own Picard variety whith the Poincare bundle equal to $\mathscr{P})$, we deduce the existence of a morphism $f: A \rightarrow A_{1}$ and of a line bundle $\mathcal{N}$ on $A$ such that

$$
\mathscr{M} \otimes p^{*}(\mathcal{N}) \simeq\left(f \times I d_{A_{1}}\right)^{*}[\mathscr{P}] .
$$

Restricting (4) on the fiber $\{x\} \times A_{1}$, for $x \in A$, one finds an isomorphism

$$
e^{*}\left(t_{x}^{*}\left(\mathcal{O}_{A}(D)\right)\right) \simeq t_{f(x)}{ }^{*}\left(\mathcal{O}_{A_{1}}\left(D_{1}\right)\right),
$$

where $e$ is the embedding $A_{1} \hookrightarrow A$. Because $D_{1}$ is a principal polarisation, the point $f(x)$ is uniquelly defined by the above property, which can be written in divisorial terms as $(\{-x\} \dot{+} D)_{\mid A_{1}}=\{-f(x)\} \dot{+} D_{1}$, at least for $x$ general such that the divisor $(\{-x\}+D)_{\mid A_{1}}$ is well defined. From this one deduces that points in $A_{1}$ are fixed by $f$ and so $f$ is surjective with $K \cap A_{1}=\left\{0_{A}\right\}$, where $K$ is the kernel of $f$.

Because $K$ cuts $A_{1}$ only in $0_{A}$, the sum morphism $K \times$ $A_{1} \rightarrow A$ is injective and so we will have $\operatorname{dim}\left(K \dot{+} D_{1}\right)=n-$ $\operatorname{dim} A_{1}+\operatorname{dim} A_{1}-1=n-1$. Now, for a general $x \in A$, we have $\{-f(x)\}+D_{1}=(\{-x\} \dot{+} D)_{\mid A_{1}} \subset\{-x\} \dot{+} D$. So, $x-f(x) \in$ $\operatorname{Tran}\left(D_{1}, D\right):=\left\{y \in A \mid\{y\}+D_{1} \subset D\right\}$. But $\operatorname{Tran}\left(D_{1}, D\right)$ is closed and so for any $x \in A$ we have $\{x-f(x)\}+D_{1} \subset D$.

Then for $x \in K,\{x\} \dot{+} D_{1} \subset D$ and therefore $K \dot{+} D_{1} \subset D$. For $K_{0}$ the connected component of the origin in $K$, we have $K_{0} \dot{+} D_{1} \subset D$. But $K_{0} \dot{+} D_{1}$ is a divisor and $D$ is prime, so the previous inclusion is an equality. Now,

$$
K_{0} \dot{+} D=K_{0} \dot{+}\left(K_{0} \dot{+} D_{1}\right)=K_{0} \dot{+} D_{1}=D \text {. }
$$

But ample $D$ implies that $K_{0}$ is finite and prime $D$ implies that $K_{0}=\left\{0_{A}\right\}$ which is equivalent with $A=A_{1}$.

Step 3. Within this step we prove that for any $i$ there is a unique $j$ such that $D_{i} \cdot E_{j} \neq 0$. For this, we consider for all $i$, an Abelian variety $B_{i}$, an ample divisor $F_{i}$ on $B_{i}$, and a surjective morphism $f_{i}: A \rightarrow B_{i}$ such that $f_{i}^{-1}\left(F_{i}\right)=D_{i}$. Their existence follows from Lemma 12.

We have

$$
n=D \cdot Z=\sum_{i=1}^{r} f_{i}^{-1}\left(F_{i}\right) \cdot Z=\sum_{i=1}^{r} F_{i} \cdot\left(f_{i}\right)_{*} Z \geq \sum_{i=1}^{r} l_{i},
$$

where $l_{i}=\operatorname{dim} B_{i}$ and the last inequality is from Proposition 9(b). We examine the last sum using the effective 
construction of $B_{i}$ 's from Lemma 12 . There, $B_{i}$ is of the form $A / K_{i}$ where $K_{i}$ is an Abelian subvariety of $A$. As consequence, $l_{i}=\operatorname{codim} K_{i}$ and so

$$
\sum_{i=1}^{r} l_{i}=\sum_{i=1}^{r} \operatorname{codim} K_{i} \geq \operatorname{codim}\left(\cap K_{i}\right)=n
$$

(by definition of $K_{i}$ and the ampleness of $D$, the intersection $\cap K_{i}$ is finite).

It results in that

$$
n \geq \sum_{i=1}^{r} F_{i} \cdot\left(f_{i}\right)_{*} Z \geq \sum_{i=1}^{r} l_{i} \geq n,
$$

and so $F_{i} \cdot\left(f_{i}\right)_{*} Z=l_{i}$. But $F_{i}$ is a prime divisor and from Step 2 there is a unique $j_{i}$ with $\left(f_{i}\right)_{*} E_{j_{i}}$ a curve. All the other curves from the support of $Z$ will be therefore contracted. We now fix $i$ and compute $D_{i} \cdot E_{j}=f_{i}^{-1}\left(F_{i}\right) \cdot E_{j}=F_{i} \cdot f_{i *} E_{j}$. This last number is 0 if $j \neq j_{i}$ and nonzero for $j=j_{i}$ because $F_{i}$ is ample. This conclude the third step.

From the first and third steps we find that $i \rightarrow j_{i}$ is a bijection and so $r=s$. Also one can reorder the curves $E_{j}$ (such that $E_{j_{i}}$ will be numbered by $E_{i}$ ) and so we can suppose that for all $i, j$ we have $D_{i} \cdot E_{j} \neq 0 \Leftrightarrow i=j$. To conclude the proof, we consider all the requirements supposed above.

In the first place we review $B_{i}$ 's. Let $T_{i}$ be the cycle $\left(f_{i}\right)_{*} Z$. From the third step, $T_{i}$ is in fact a curve, namely, $f_{i}\left(E_{i}\right)$. Also we have seen that $F_{i} \cdot T_{i}=l_{i}=\operatorname{dim} B_{i}$ and therefore Theorem 11 implies that $B_{i}$ is the Jacobian of $T_{i}$. To see this, we need only to prove that $T_{i}$ is a generating curve of $B_{i}$ and this is implied by the fact that, as we have seen, $f_{i}$ contracts all the curves $E_{j}$ for $j \neq i$ and as far as these contain $0_{A}$, the contraction will be to $0_{B_{i}}$. So $f_{i}(A)=f_{i}\left(A_{i}\right)=B_{i}$ and because $E_{i}$ generates $A_{i}$, $T_{i}$ generates $B_{i}$. So, by Theorem $11, F_{i}$ is a translation of the canonical divisor on $B_{i}$.

Let us recall that in the first step we supposed (using appropriate translations) that all $D_{i}$ 's cut properly the subvarieties $A_{j}$ 's, which means that either $e_{j}{ }^{*}\left(D_{i}\right)$ is an effective divisor on $A_{j}$ or $D_{i} \cap A_{j}$ is empty, in which case $e_{j}{ }^{*}\left(D_{i}\right)=0$. The former case can happen only for $j=i$, because in this situation $e_{j}{ }^{*}\left(D_{i}\right) \cdot E_{j} \neq 0$ (more precisely, the projection formula gives $\left.e_{j}{ }^{*}\left(D_{i}\right) \cdot E_{j}=D_{i} \cdot e_{j_{*}} E_{j}=D_{i} \cdot E_{j}\right)$. So $e_{j}{ }^{*}\left(D_{i}\right) \neq 0 \Leftrightarrow j=i$ and we have

$$
\begin{aligned}
D_{j}^{\prime} & =e_{j}^{*}(D)=e_{j}^{*}\left(D_{j}\right)=e_{j}^{*} f_{j}^{*}\left(F_{j}\right) \\
& =\left(f_{j} \circ e_{j}\right)^{*}\left(F_{j}\right) .
\end{aligned}
$$

Let us consider the morphism $f_{j} \circ e_{j}: A_{j} \rightarrow B_{j}$. It sends the generating curve $E_{j}$ of $A_{j}$ on the generating curve $T_{j}$ of $B_{j}$, and therefore it is surjective; so $d_{j} \geq l_{j}$. But, from the first and third steps, $n=\sum_{j=1}^{r} d_{j}=\sum_{j=1}^{r} l_{j}$; this implies that $f_{j} \circ e_{j}$ has a finite nonzero degree. On the other hand $f_{j} \circ e_{j}$ pull back the principal polarization $F_{j}$ from $B_{j}$ to the principal polarization $D_{j}^{\prime}$ on $A_{j}$. So its degree is 1 and it is an isomorphism with inverse denoted by $g_{j}$.

Let $h: B_{1} \times \cdots \times B_{r} \rightarrow A$ be defined by $h\left(b_{1}, \ldots, b_{r}\right)=$ $\sum_{i=1}^{r} g_{i}\left(b_{i}\right)$ and $g: A \rightarrow B_{1} \times \cdots \times B_{r}$ be defined by $g(a)=\left(f_{1}(a), \ldots, f_{r}(a)\right)$. Then $h \circ g$ is the identity, being the identity on every $A_{i}$. Also, $g \circ h$ is the identity, being the identity on every $\left\{0_{B_{1}}\right\} \times \cdots \times B_{i} \times \cdots \times\left\{0_{B_{r}}\right\}$.

So $h$ is an isomorphism, $B_{i}$ is the Jacobian of $T_{i}$, and the last part of the theorem concerning the form of the divisors $D_{i}$ and curves $T_{i}$ is obvious due to the fact that the transformations of $D_{i}$ and $T_{i}$ were translations.

Finally, we formulate the following corollary which is the result of Hoyt from [2].

Corollary 14. Let $A$ be an Abelian variety, $D$ an ample divisor with $D^{n}=n !$, and $Z$ a 1-cycle such that $D^{n-1}$ is numerically equivalent with $(n-1) ! Z$. Then the conclusion of Theorem 13 holds true.

Proof. We have $D^{n}=(n-1) ! D \cdot Z \cdot$, so $D \cdot Z=n$. On the other hand, from Proposition $6(c),(n-1) ! Z$ is a generating 1-cycle and therefore $Z$ is a generating 1-cycle. Now everything is a consequence of Theorem 13.

\section{Conflict of Interests}

The authors declare that there is no conflict of interests regarding the publication of this paper.

\section{References}

[1] I. Matsusaka, "On a characterization of a Jacobian variety," Memoirs of the College of Science, University of Kyoto, Series A: Mathematics, vol. 32, pp. 1-19, 1959.

[2] W. L. Hoyt, "On products and algebraic families of Jacobian Varieties," The Annals of Mathematics, vol. 77, no. 3, pp. 415-423, 1963.

[3] Z. Ran, "On subvarieties of abelian varieties," Inventiones Mathematicae, vol. 62, no. 3, pp. 459-479, 1980.

[4] N. Buruiana, "Algebraic cycles on symmetric products and Abelian varieties," Revue Roumaine de Mathématique Pures et Appliquées, vol. 45, no. 4, pp. 593-608, 2000.

[5] A. Collino, "A new proof of the Ran-Matsusaka criterion for Jacobians," Proceedings of the American Mathematical Society, vol. 92, no. 3, pp. 329-331, 1984.

[6] S. Lang, Abelian Varieties, Interscience Tracts in Pure and Applied Mathematics, vol. 7, 1959.

[7] D. Mumford, Abelian Varieties, Oxford University Press, 1970.

[8] I. Matsusaka, "On a generating curve of an Abelian variety," Natural Science Report of the Ochanomizu University, vol. 3, pp. $1-4,1952$. 


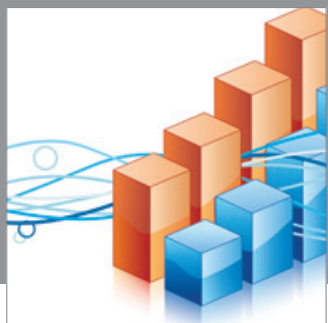

Advances in

Operations Research

mansans

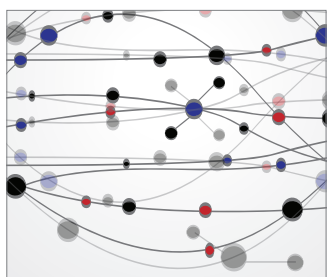

The Scientific World Journal
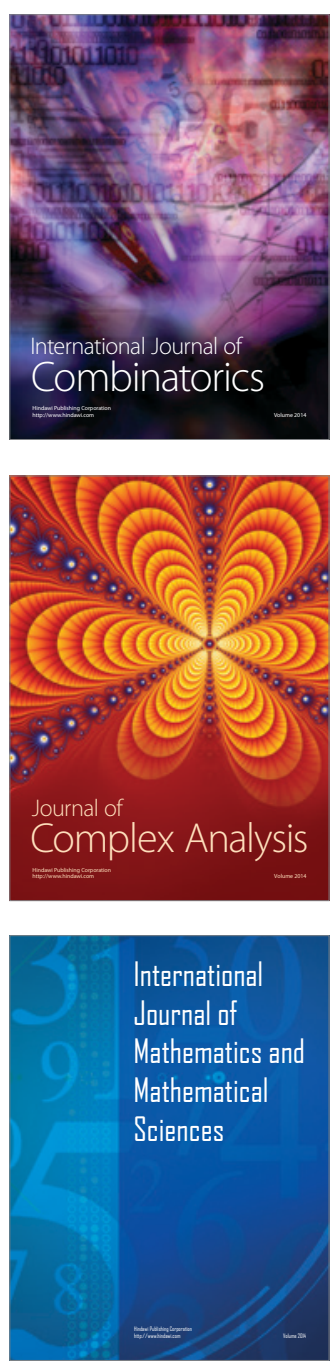
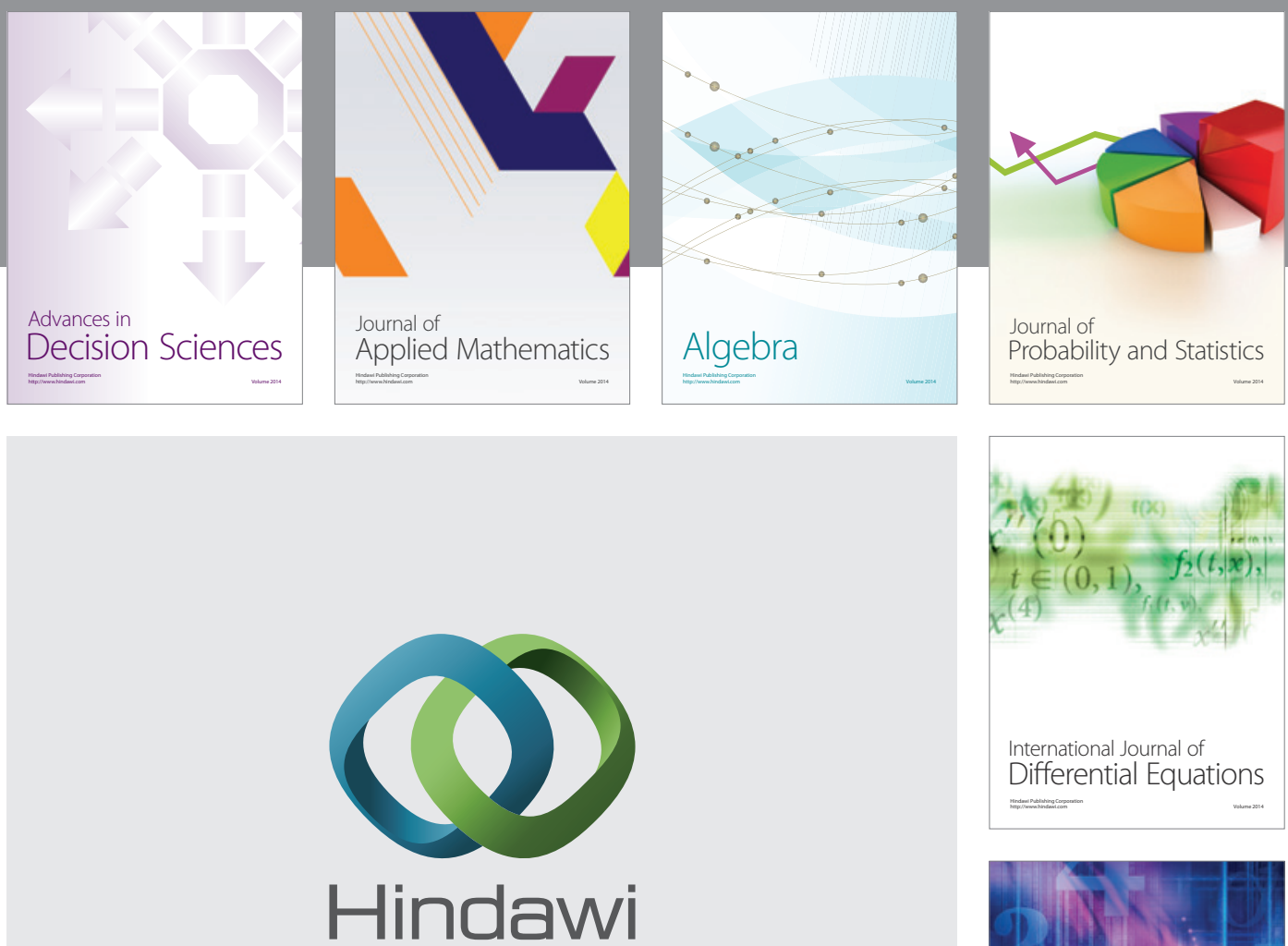

Submit your manuscripts at http://www.hindawi.com
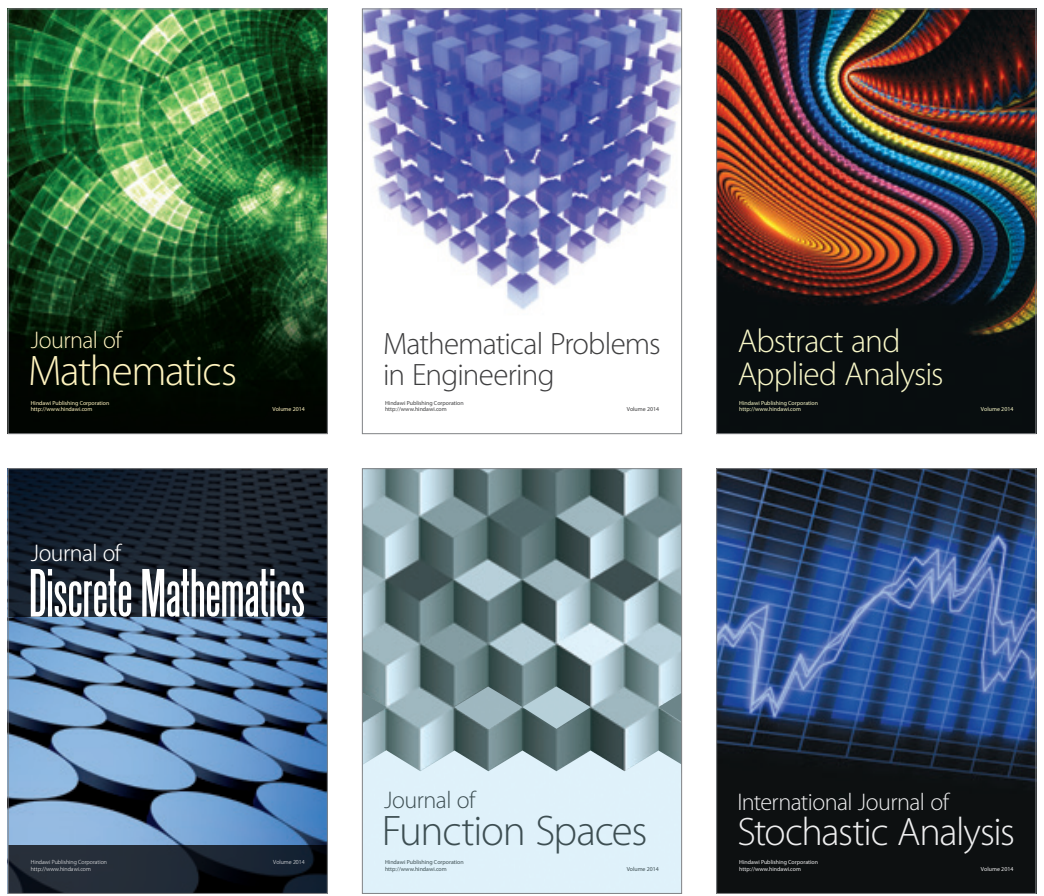

Journal of

Function Spaces

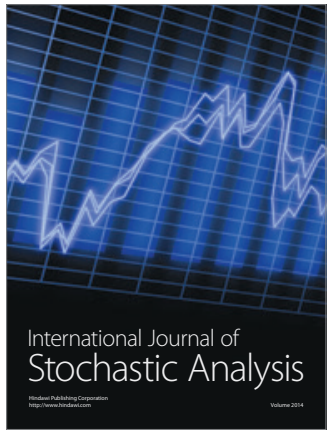

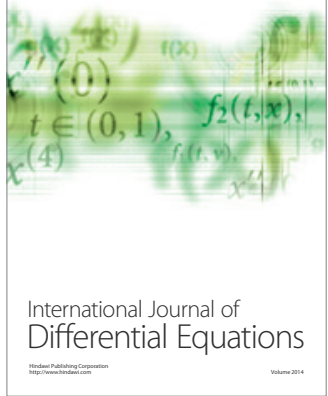
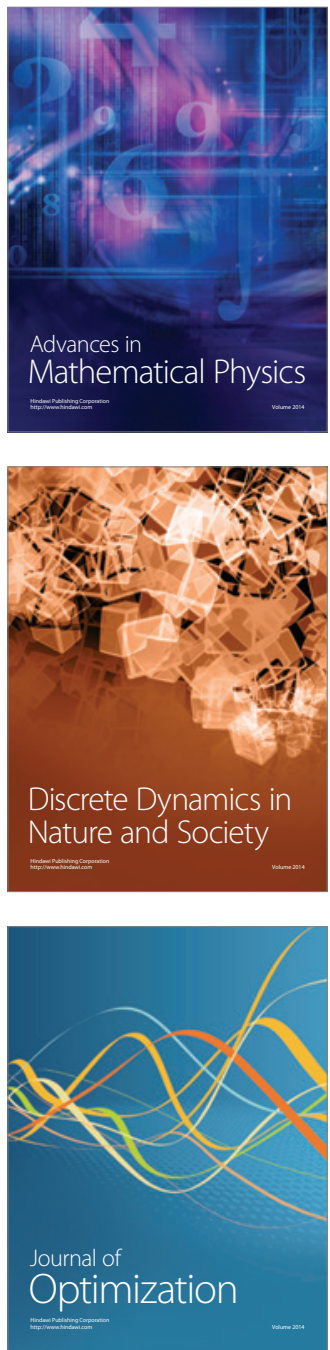